ORIGINAL ARTICLE

\title{
The right place in the right space? Awareness of site for needle thoracocentesis
}

\author{
E P Ferrie, N Collum, S McGovern
}

Emerg Med J 2005;22:788-789. doi: 10.1136/emj.2004.015107

See end of article for authors' affiliations

......................

Correspondence to: Eoghan P Ferrie, Ulster Hospital, Dundonald, A\&E department, Northern Ireland; eoghanferrie@ hotmail.com

Accepted for publication 22 December 2004

\begin{abstract}
Background: Invasive practical procedures require identification of surface anatomical landmarks to reduce risk of damage to other structures. Needle thoracocentesis has specific complications, which have been previously documented. An observational study was performed among emergency physicians to name the landmark for needle thoracocentesis and identify this point on a human volunteer as per Advanced Trauma and Life Support (ATLS) guidelines. Results: A cohort of 25 emergency physicians was studied, 21 (84\%) of which were ATLS certified. The correct landmark was named by 22 (88\%). Only 15 $(60 \%)$ correctly identified the second intercostal space on the human volunteer, all placing the needle medial to the midclavicular line, with a range of $3 \mathrm{~cm}$. Two (8\%) named and identified the site of needle pericardiocentesis; one (4\%) named and identified the fifth intercostal space in the anterior axillary line. Discussion: These results demonstrate a low accuracy among emergency physicians in identifying correct landmarks for needle thoracocentesis under elective conditions. Should greater emphasis be placed on competency based training in ATLS?
\end{abstract}

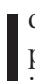
dentification of regional surface anatomy is essential when performing invasive procedures to minimise likelihood of iatrogenic injury.

Needle thoracocentesis is a life saving procedure, which involves placing a wide-bore cannula into the second intercostal space midclavicular line (2ICS MCL), just above the third rib, in order to decompress a tension pneumothorax, as per Advanced Trauma Life Support (ATLS) guidelines. ${ }^{1}$ Surrounding this landmark are the mediastinal structures and the internal mammary artery medially, and the subclavian vessels and subcostal neurovascular bundles superiorly. There have been several case reports of life threatening iatrogenic injury following laceration of these structures during needle thoracocentesis. ${ }^{2-5}$

It is well documented that there are different attrition rates for knowledge and skill. ${ }^{6-11}$

We hypothesise that there is a discrepancy between knowledge of, and ability to identify, the site for placement of the decompressing needle.

\section{METHODS}

An observational study was performed in order to investigate the knowledge and skills in identifying surface landmarks for needle thoracocentesis amongst emergency physicians. The grade of doctor and ATLS status were recorded. Each participant was instructed to name the correct landmark for needle thoracocentesis and then asked to identify the site with a water soluble pen mark on a male human volunteer. The pen mark was transferred to a transparent sheet lined up against predetermined marker points on the volunteer's chest. The pen mark was then sequentially removed.

At the end of the data collection period, the correct landmark was identified using measuring tape to identify the midclavicular line; both palpation and ultrasound were used to identify the ribs and intercostal spaces. The point thus identified as the middle of the second intercostal space, midclavicular line was independently verified by two of the authors and transferred to a transparent sheet in the same way as before, which served as a template. The template was placed over each participant's transparent sheet in turn, and the distance between the two points measured. Two authors independently measured these distances and any disparity was re-measured and the mean result taken.

\section{RESULTS}

Twenty five emergency physicians were included: six senior house officers, five staff grades, six specialist registrars, and eight consultants. Twenty two of the participants had completed ATLS training within the previous 10 years. The other four were senior house officers who had no formal ATLS teaching but were assumed to be aware of the procedure given the speciality they were working in.

Twenty two (88\%, with 95\% confidence interval (CI) 69 to $95 \%$ ) of the participants named the landmark as 2ICS MCL. Fifteen of the 25 (60\%, with $95 \%$ CI 39 to $79 \%)$ were able to correctly identify the second intercostal space on the volunteer. Four (16\%) incorrectly identified the first intercostal space and four (16\%) identified the third intercostal space. Altogether, 21 of the $22(95 \%)$ points were placed medial to the midclavicular line. One $(4 \%)$ named and identified the fifth intercostal space, anterior axillary line and two $(8 \%)$ named and identified "below and lateral to the xiphisternum" as the landmark.

Of the 14 who correctly identified the second intercostal space, $12(86 \%)$ were positioned in the lower half of the intercostal space.

Following statistical analysis with two sided $\chi^{2}$ tests and Fishers exact test, there was no significant difference between correct identification of the landmark and seniority of participant, nor correct identification and ATLS status. Because of the small sample size, however, this study would not have been sufficiently powered to fully explore these secondary end points. Results are summarised in fig 1 .

\section{DISCUSSION}

The primary aim of this study was to describe the relation between knowledge of the preferred site of needle thoracocentesis and the ability to correctly identify this point on a human volunteer.

Abbreviations: ATLS, Advanced Trauma Life Support 


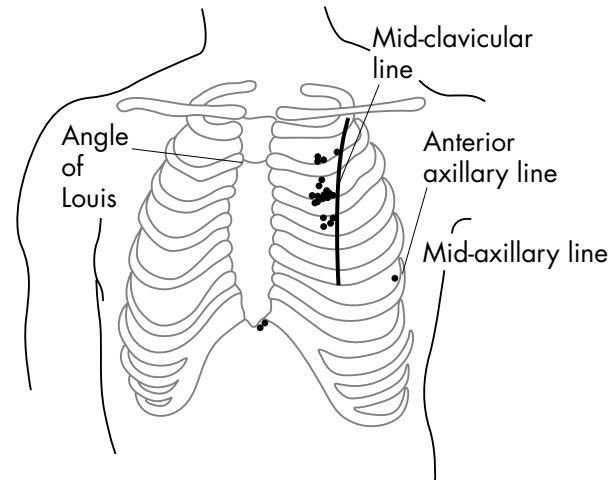

Figure 1 Diagram showing range of points positioned on chest. All the marks for 2ICS MCL were positioned medially.

Previous case reports confirm the dangers of bleeding secondary to iatrogenic injury. ${ }^{2-5}$ Erroneous medial placement of the needle increases the risk of damage to the internal mammary vessels and superior mediastinal structures. The reasons for erroneous medial placement of the needle are unclear, but the authors suggest a failure to identify the lateral margin of the clavicle with subsequent underestimation of its length may be contributory.

The majority, $22 / 25$ (88\%), of emergency physicians in the study group could name the preferred site of needle placement; however, only 15/25 (60\%) could correctly identify the 2ICS MCL on a human volunteer under elective conditions. This represents a large disparity between theoretical knowledge and hands-on skills. Could this be a reflection of the format of teaching or does this simply represent the attrition rate of integrated but non-automated skills?

The two physicians who named and placed their needle point below and lateral to the xiphisternum may have misinterpreted the written instruction to "identify the site for needle thoracocentesis" as "identify the site for needle pericardiocentesis". This represents a basic error in the ability to follow instructions, although we cannot speculate that either of these participants would have correctly identified the site for needle thoracocentesis if this error had been pointed out.

The physician who named and placed their needle point in the fifth intercostal space in the anterior axillary line correctly identified an alternate site for placement of the needle, ${ }^{2}$ although this is not currently within the ATLS guidelines. There have been reports where decompression through 2ICS MCL has failed to release a tension pneumothorax, possibly because of insufficient cannula length, ${ }^{6-9}$ and it has been postulated that the fifth intercostal space, anterior axillary line may be preferable as it avoids the bulky anterior chest wall muscles. ${ }^{6}$ However, there may be a higher complication rate with this site as pleural adhesions are more likely to be present in the lower chest cavity. ${ }^{10}$

The assessment technique used in this study was designed as simple, rapid, and reproducible. We have not formally validated our study method by external review, but no satisfactory alternative was found within the literature.
One of the limitations of this study was the small sample size, from which it was difficult to draw statistical significance, although it highlights important issues that impact on clinical practice. The use of a single human volunteer was an attempt to standardise the testing procedure; however, a range of volunteers may have given more useful results.

Needle thoracocentesis for decompression of a tension pneumothorax is life saving, but is associated with potentially serious complications. Care must be taken in identifying surface landmarks to minimise the risk involved. A greater emphasis on competency based training should assist in achieving this.

\section{Authors' affiliations}

E P Ferrie, N Collum, S McGovern, Ulster Hospital Dundonald, Ulster, Northern Ireland

Competing interests: none declared

"The Corresponding Author has the right to grant on behalf of all authors and does grant on behalf of all authors, an exclusive licence (or non exclusive for government employees) on a worldwide basis to the BMJ Publishing Group Ltd to permit this article (if accepted) to be published in EMJ and any other BMJPGL products and sublicences such use and exploit all subsidiary rights, as set out in our licence (http:// emi.bmijournals.com/misc/ifora/licenceform.shtml)."

\section{REFERENCES}

1 American College of Surgeons. Advanced Trauma Life Support, 6th edition. Chicago, IL, 1997.

2 Rawlins R, Brown KM, Carr CS, et al. Life threatening haemorrhage after anterior needle aspiration of pneumothoraces. A role for lateral needle aspiration in emergency decompression of spontaneous pneumothorax. Emerg Med J 2003;20:383-4.

3 Seneff MG, Corwin RW, Gold LH, et al. Complications associated with thoracocentesis. Chest 1986;90:97-100.

4 Carney M, Ravin CE. Intercostal artery laceration during thoracocentesis. Chest 1979;75:520-2.

5 Butler KL, Best IM, Weaver WL, et al. Pulmonary artery injury and cardiac tamponade after needle decompression of a suspected tension pneumothorax. J Trauma 2003;54:610-11.

6 Fossel M, Kiskaddon RT, Sternbach GL. Retention of cardiopulmonary resuscitation skills by medical students. J Med Educ 1983;58(7):568-75.

7 Mancini ME, Kaye W. The effect of time since training on house officers' retention of cardiopulmonary resuscitation skills. Am J Emerg Med 1985;3(1):31-2.

8 Young R, King L. An evaluation of knowledge and skill retention following an in-house advanced life support course. Nurs Crit Care 2000;5(1):7-14.

9 Hammond F, Saba M, Simes T, et al. Advanced life support: retention of registered nurses' knowledge 18 months after initial training. Aust Crit Care 2000;13(3):99-104

10 Ali J, Howard M, Williams J. Is attrition of advanced trauma life support acquired skills affected by trauma patient volume? Am J Surg 2002; 183(2): 142-5

11 Ali J, Howard M, Williams JI. Do factors other than trauma volume affect attrition of ATLS-acquired skills? J Trauma 2003;54(5):835-41.

12 Jenkins C. Needle thoracocentesis fails to diagnose a large pneumothorax. Anaesthesia 2000;55:925-6.

13 Britten S, Palmer S. Chest wall thickness may limit adequate drainage of tension pneumothorax by needle thoracocentesis. J Accid Emerg Med 1996; 13:426-7.

14 Britten S, Palmer SH, Snow TM. Needle thoracocentesis in tension pneumothorax: insufficient cannula length and potential failure. Injury 1996;27:321-2.

15 Hostetler MA, Davis CO. Bilateral localized tension pneumothoraces refractory to needle decompression. Pediatr Emerg Care 1999;15:322-4.

16 Goodman LR, Putman CE. Intensive care radiology. Imaging of the Critically III. 2nd edition. Philadephia: WB Saunders, 1982:99-100. 